\title{
Time-lapse Microscopy of Fusarium oxysporum-Arabidposis thaliana root interactions
}

\author{
D.H. Powell*, K.J. Czymmek*, and S. Kang** \\ *University of Delaware, Delaware Biotechnology Institute, Newark, DE 19711 USA \\ **Penn State University, Department of Plant Pathology, University Park, PA 16802 USA
}

Although historically a number of strategies have been employed to clearly discriminate fungal structures within diseased plant tissues using light microscopy, there are a number of difficulties associated with this undertaking. The most daunting aspects are the relatively impermeable and chemically variable plant and fungal cell walls and the lack of robust contrasting methods for fungal entities. With the advent of fluorescent proteins, the aforementioned limitations have been virtually eliminated and their utility repeatedly demonstrated as vital markers for documenting in planta plant-pathogen interactions [1].

However, root pathogens are especially challenging to image in vivo due to the opacity of soil, which prevents unperturbed and unobstructed observation. To circumvent this, our lab developed an in vivo agar-based method for observation of pathogenic root interactions between Fusarium oxysporum, the causal agent of vascular wilt disease, and Arabidopsis thaliana, a flowering plant from the mustard family [2]. As an extension to this agar-based approach we have developed a soil-based root pathogenesis system for time-lapse confocal microscopy. To image fungal ingress we targeted ZsGreen constitutively expressed in the cytoplasm of $F$. oxysporum. A. thaliana was grown in soil supplemented with vitamins [3] until roots were growing along the surface of a chamber with a coverslip bottom. Chambers were subsequently inoculated with ZsGreen expressing conidia of $F$. oxysporum and monitored during disease progression.

Time-lapse acquisition of infection events in roots of plants was documented in three-dimensions using confocal microscopy. This approach confirmed results from our agar-based system that fungal invasion involved penetration at primary root apices and at lateral root initials (not shown). More importantly, we were able to document this host-pathogen interaction in what was considered a more natural soil environment (Figs. 1A-D). Although the presence of opaque soil particles around the plant prevented the visualization of plant-fungal interactions in their entirety, a substantial degree was unobstructed for documentation. Details including initial contact, penetration, and vascular progression were readily observed from individual encounter sites. Simple modifications of this method for dynamic imaging of plant-pathogen interactions within the soil could be applied toward numerous rhizosphere related investigations where artificial media is unsuitable.

References

[1] K.J. Czymmek et al., Mycologia 94 (2002) 280

[2] K.J. Czymmek et al., Microsc. Microanal. 10 (Suppl 2) (2004) 216

[3] T. Murashige and F. Skoog, Physiol. Plant. 15 (1962) 473 

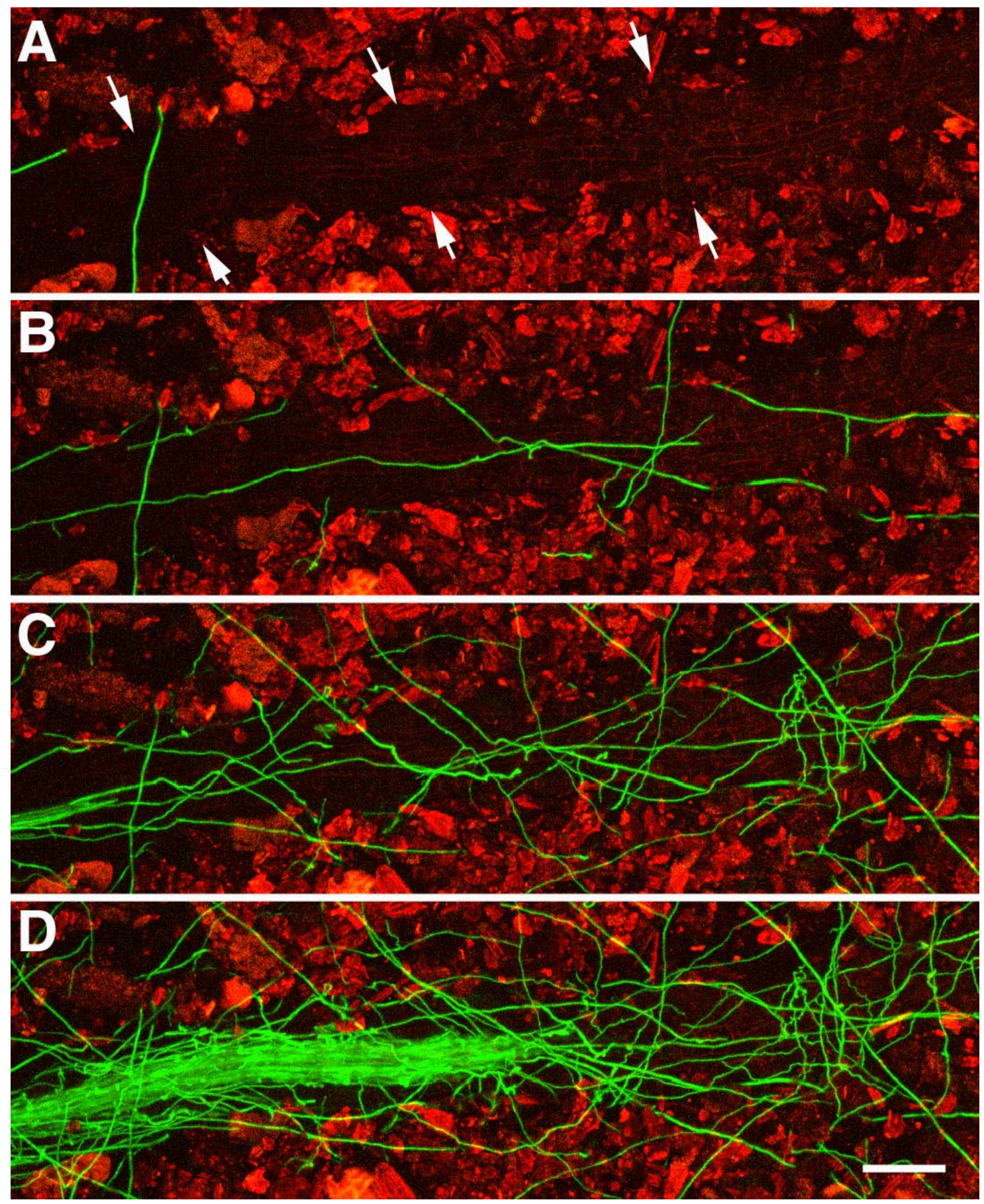

Figures 1A-D. In vivo confocal microscopy of Arabidopsis thaliana-Fusarium oxysporum hostpathogen interactions in soil. Fig. 1A. Early infection or root (dark zone between arrows) in soil (red) involved initial contact by ZsGreen expressing hyphae of $F$. oxysporum (green). ( $\mathrm{T}=0 \mathrm{hr}$ ). Fig. 1B. Initially, hyphal growth was primarily linear along the long axis of the root. $(\mathrm{T}=10 \mathrm{hr})$. Fig. $1 \mathrm{C}$. Once the root tip was penetrated (not shown - root tip on left) hyphal growth in nearby, distil root regions became more branched and irregular. $(\mathrm{T}=31 \mathrm{hr})$. Fig.1D. Vascular ramification from the root tip proceeded in a rapid and massive fashion. $(\mathrm{T}=40 \mathrm{hr})$. Bar $=100 \mu \mathrm{m}$. 